\begin{tabular}{|r|l|}
\hline \multicolumn{2}{|c|}{ Statistica Sinica Preprint No: SS-2020-0330 } \\
\hline Title & $\begin{array}{l}\text { Distributed Empirical Likelihood Approach to Integrating } \\
\text { Unbalanced Datasets }\end{array}$ \\
\hline Manuscript ID & SS-2020-0330 \\
\hline URL & http://www.stat.sinica.edu.tw/statistica/ \\
\hline DOI & $10.5705 /$ ss.202020.0330 \\
\hline Complete List of Authors & $\begin{array}{l}\text { Ling Zhou, } \\
\text { Xichen She and } \\
\end{array}$ \\
Peter X.-K. Song \\
\hline Corresponding Author & Peter X.-K. Song \\
\hline E-mail & pxsong@umich.edu \\
\hline Notice: Accepted version subject to English editing. \\
\hline
\end{tabular}




\title{
Distributed Empirical Likelihood Approach to Integrating Unbalanced Datasets
}

\author{
Ling ZHOU, Xichen SHE and Peter X.-K. SONG \\ Southwestern University of Finance and Economics \\ University of Michigan
}

Abstract: This paper proposes a distributed empirical likelihood (DEL) method to perform an integrative analysis of multiple data sources with the flexibility on handling either homogeneous or heterogeneous data. The proposed DEL method does not require pooling individual datasets into a centralized operational platform, so the privacy of subject-level information in individual data sources is protected. The DEL method is shown almost surely equal to the centralized empirical likelihood approach that would be adopted if individual datasets were combined and stored at one place. Key large-sample properties and algorithm convergence of the DEL method are established. The numerical performance of the DEL method is illustrated by simulation studies and a real data example in which the DEL method appears clearly advantageous over the classical meta-estimation method in the analysis of unbalanced datasets.

Key words and phrases: ADMM, Data Privacy, Data Integration, Divide-and- 
Conquer, Meta Estimation.

\section{Introduction}

One of the primary tasks in data integration aims to combine data from different sources in order to perform analysis in a comprehensive and unified manner, which can overcome limitations in separate analyses of individual data sources (Lenzerini 2002, Halevy et al. 2006). For example, consider a situation in which one data source contains observations collected from students in elementary schools, while another source contains measurements from students in middle schools. These two data sources present different ranges of age, and merging them can unveil statistics and conclusions generalizable to a broader population than possible with an analysis of a single data source. In many applications, appropriately integrating multiple data sources enables practitioners to empower their data analysis to answer a scientific hypothesis of interest Citro 2014, Lohr \& Raghunathan 2017, National Academies of Sciences Engineering and Medicine 2017). Proposed first by Owen (1988, 1991), the empirical likelihood (EL) method is one of primary statistical methods for estimation and inference, due to some methodological advantages over other existing methods. For example, the EL method requires minimal parametric model assumptions for data distri- 
butions, allows for the construction of data-driven confidence regions, and enjoys the ease of handling auxiliary information. Such properties are particularly appealing in the operation of data integration. See Owen (2001) for a comprehensive overview on the EL method, and more references therein.

The focus of this paper is to develop a distributed EL method (DEL) for an integrative data analysis when multiple sources of subject-level data are accessible at local sites but combining individual datasets is prohibited. Specifically, consider $K$ independently sampled datasets from $K$ study sites, $\boldsymbol{W}^{[k]}=\left\{\boldsymbol{W}_{k i}\right\}_{i=1}^{n_{k}}, k=1, \ldots, K$, with the $k^{t h}$ dataset consisting of $n_{k}$ i.i.d. observations. To analyze the $k^{\text {th }}$ dataset alone, we estimate a $(q+1)$-dimensional parameter $\boldsymbol{\theta}_{k 0} \in \Xi \subset \mathcal{R}^{q+1}$ through the following set of moment conditions: $E\left\{g_{k}\left(\boldsymbol{W}_{k i} ; \boldsymbol{\theta}_{k 0}\right)\right\}=\mathbf{0}$, for $i=1, \cdots, n_{k}, k=$ $1, \cdots, K$, where $g_{k}\left(\boldsymbol{W}_{k i} ; \boldsymbol{\theta}\right)$ is an $m_{k}$-dimensional estimating function with $m_{k} \geq(q+1)$. In this paper, we consider two scenarios of integrative analyses of practical importance: (a) homogeneity case: both parameters and estimating functions are assumed the same for all $K$ data sources, namely $\boldsymbol{\theta}_{k 0} \equiv \boldsymbol{\theta}_{0}$ and $g_{k}(\cdot) \equiv g(\cdot)$ for all $k$; and (b) partial homogeneity case: $\boldsymbol{\theta}_{k 0} \equiv \boldsymbol{\theta}_{0}$, while moment conditions $g_{k}(\cdot)$ 's may be different for $k$. In effect, scenario (a) is typically considered in the classical meta-analysis that requires data distributions in individual data sources are reasonably 
balanced, so that reliable site-specific statistics may be obtained prior to a meta-estimation. However, this individual balance may fail to hold in practice. In the example of the USA kidney transplant data, three binary covariates on transplant recipients, obesity, previous transplant, and hepatitis $\mathrm{C}$ serology in region Guam, take the same value with no variability (i.e. zero variances). In this case, the classical meta-type method fails, while our proposed DEL method can work properly because the DEL method aggregates estimating functions across study sites whereas the meta-estimation directly aggregates site-specific summary statistics.

There is little work available in the literature concerning the EL methodology in integrative data analyses. Most of published works have focused on improving the EL method under a single dataset by incorporating auxiliary information. For example, Chen \& Kim (2014) developed an EL method for a finite population, incorporating features of sampling design via suitable constraints. Han \& Lawless (2019) studied an EL estimation using a certain summary of auxiliary information for efficiency improvement. Along this line of research, Huang et al. (2016) proposed a double EL method to estimate survival time distribution by synthesizing individual-level data from an external data source. See also Chen \& Qin (1993), Qin (2000), Chaudhuri et al. (2008), Qin et al. (2014), among others. However, the objective 
of an integrative data analysis differs from the aforementioned existing approaches; it pertains to a joint inference with multiple data sources, in which each dataset is regarded as a primary information source and treated equally in the distributed estimation.

One straightforward solution to data integration is the so-called centralized method; that is, all datasets are combined together, stored, and processed in a central computing facility. Consequently, the EL method may be directly applied to analyze the combined data. The feasibility of such approach depends obviously on the availability of combined data, which can be challenged by limitations of computing facility, data use agreement and data privacy consideration. Data privacy is one of the biggest barriers for data sharing and data combination. For example, although sharing patients' health records across hospitals is beneficial for medical research, this process takes significant amounts of administrative effort and time, and thus is practically very tedious, and subject to information censoring because of concerns on data privacy and regulatory policies of data sharing. In addition, the computational burden may become very substantial with rapidly increased volumes of merged data. For the swarms of omics data, imaging data, and mobile health data generated by modern high-throughput technologies, storing and processing all the data at one single computing 
facility may be prohibited, so divide-and-conquer strategy via distributed computing becomes a popular solution in practice. It is interesting to note that the centralized data analysis may be less efficient or even invalid when there exist some substantial data heterogeneity across data sources.

Being a well-known approach, the meta-analysis combines site-specific summary statistics (Simmonds \& Higgins 2007, Kovalchik 2012). It takes a weighted average of individual summary statistics to produce an overall estimator for a common population attribute $\boldsymbol{\theta}_{0}$ of interest:

$$
\hat{\boldsymbol{\theta}}_{\text {meta }}=\left(\sum_{k=1}^{K} V_{k}^{-1}\right)^{-1}\left(\sum_{k=1}^{K} V_{k}^{-1} \hat{\boldsymbol{\theta}}_{k}\right),
$$

where $\hat{\boldsymbol{\theta}}_{k}$ is, say, an EL estimate obtained from the $k^{\text {th }}$ data source, and $V_{k}=\operatorname{Var}\left(\hat{\boldsymbol{\theta}}_{k}\right)$ is the corresponding variance. Having no closed-form expression, an approximate variance $V_{k}$ is given for a large $n_{k}$; see Qin \& Lawless (1994). The validity of this meta-estimation in (1.1) may be easily justified where all $n_{k}$ are large by the means of confidence distribution method first proposed by Efron (1993) and later advocated by Xie \& Singh (2013). The large-sample behavior may derail with finite sample sizes; for example, in practice that some data sources often have low sample sizes, for which the approximate variances are no longer reliable, so the resulting meta estimator in (1.1) may perform poorly. A possible remedy to improve the estimation of $V_{k}$ may be done by a resampling method, which incurs 
excessive computational costs. Furthermore, some variables may show unbalanced distributions when sample sizes are small across data sources (e.g. measurements of a covariate having little variability). In this case, the meta-estimation method can fail.

In this paper we investigate a new approach to distributed computing

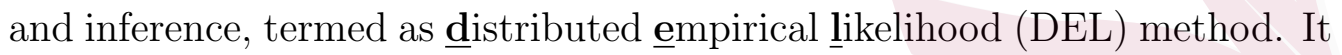
performs EL estimation and inference without pooling individual datasets together or sharing subject-level information across datasets. To address the two scenarios (a) and (b), we present two variants of DEL methods: (i) doubly constrained (DOC) DEL (DEL.DOC) method, suitable for the homogeneity scenario (a); (ii) singly constrained (SIC) DEL (DEL.SIC) method, applicable to the partial homogeneity scenario (b). In comparison to the conventional meta-estimation method, our new contributions of the DEL methodology are four-fold: (i) In both scenarios, our DEL method still works in the presence of unbalanced distributions of variables across multiple data sources where the meta-type method fails. (ii) In scenario (a), the DEL.DOC estimator is asymptotically equivalent to that obtained by the centralized method (termed as the centralized EL estimator (CEL) hereon) in the sense of "almost surely" (Stout 1974); that is, DEL.DOC method produces an EL estimator that is equal, almost surely, to the CEL 
estimator. In contrast, the meta-type estimator in (1.1) is only weakly equivalent to the CEL estimator in the sense of "in distribution" (Liu et al. 2015). (iii) DEL.DOC method provides a valid inference not only under a fixed $K$ but also under the case of $K$ diverging to infinity at any rate. (iv) Neither DEL.DOC method nor DEL.SIC method requires a direct estimation of individual variances $V_{k}$ in the operation of DEL method.

The rest of the paper is organized as follows. Section 2 introduces necessary notations and then presents DEL.DOC method under the homogeneity scenario (a). Extension of DEL method to the partial homogeneity scenario (b) is discussed in Section 3, where theoretical properties of DEL.SIC method are investigated. Simulation studies and a real data example for the evaluation of the two proposed methods are reported in Sections 4 and 5 , respectively. Section 6 contains some concluding remarks. All technical details can be found in the supplementary materials.

\section{DEL method in the homogeneity setting}

Under scenario (a) of parameters $\boldsymbol{\theta}_{k 0} \equiv \boldsymbol{\theta}_{0}$ and estimating functions $g_{k}(\cdot) \equiv$ $g(\cdot)$ for all $k$, we present the doubly constrained DEL (DEL.DOC) method after a brief review of the centralized EL (CEL)method. 


\subsection{Centralized EL method9}

\subsection{Centralized EL method}

Consider the $k^{\text {th }}$ dataset $\boldsymbol{W}^{[k]}$ of $n_{k}$ i.i.d. observations. The parameter of interest $\boldsymbol{\theta}_{0} \in \boldsymbol{\Xi}$ satisfies the mean-zero moment condition: $E_{\boldsymbol{\theta}_{0}}\left\{g\left(\boldsymbol{W}_{k i} ; \boldsymbol{\theta}_{0}\right)\right\}=$ 0. Here $\boldsymbol{\Xi} \subset \mathcal{R}^{q+1}$ is a compact set containing $\boldsymbol{\theta}_{0}$ as an interior point. According to the theory of empirical likelihood (Owen 1988, Qin \& Lawless 1994), parameter $\boldsymbol{\theta}_{0}$ may be estimated by maximizing the following objective function based on dataset $\boldsymbol{W}^{[k]}: L_{k}(\boldsymbol{\theta})=\sup _{p_{k i}} \prod_{i=1}^{n_{k}} n_{k} p_{k i}$, subject to $p_{k i} \geq 0, i=1, \cdots, n_{k}, \sum_{i=1}^{n_{k}} p_{k i}=1, \sum_{i=1}^{n_{k}} p_{k i} g\left(\boldsymbol{W}_{k i} ; \boldsymbol{\theta}\right)=\mathbf{0}$. The EL estimator $\hat{\boldsymbol{\theta}}_{k}=\arg \max _{\boldsymbol{\theta} \in \boldsymbol{\Xi}} L_{k}(\boldsymbol{\theta})$. For each $\boldsymbol{\theta} \in \boldsymbol{\Xi}$, let $\mathcal{T}_{n_{k}}(\boldsymbol{\theta})=$ $\cap_{i=1}^{n_{k}}\left\{\boldsymbol{t}: \boldsymbol{t}^{T} g_{k i}(\boldsymbol{\theta})<1\right\}$, where $g_{k i}(\boldsymbol{\theta})=g\left(\boldsymbol{W}_{k i} ; \boldsymbol{\theta}\right)$. Denote $h_{k}(\boldsymbol{\theta}, \boldsymbol{t})=$ $n_{k}^{-1} \sum_{i=1}^{n_{k}} \log \left(1-\boldsymbol{t}^{T} g_{k i}(\boldsymbol{\theta})\right)$. It is known that $\hat{\boldsymbol{\theta}}_{k}$ may be obtained by solving the saddle-point problem:

$$
\hat{\boldsymbol{\theta}}_{k}=\underset{\boldsymbol{\theta} \in \boldsymbol{\Xi}}{\arg \min } \sup _{\boldsymbol{t} \in \mathcal{T}_{n_{k}}(\boldsymbol{\theta})} h_{k}(\boldsymbol{\theta}, \boldsymbol{t})
$$

When multiple data sources $\boldsymbol{W}^{[k]}, k=1, \ldots, K$, are available, as long as the capacity of a computing facility permits, a centralized EL (CEL) method can be done by first aggregating all datasets on a centralized computing site, and then acquiring an estimator $\hat{\boldsymbol{\theta}}_{c e n}$ of the form:

$$
\hat{\boldsymbol{\theta}}_{\text {cen }}=\underset{\boldsymbol{\theta} \in \boldsymbol{\Xi}}{\arg \min } \sup _{\boldsymbol{t} \in \mathcal{T}_{n}(\boldsymbol{\theta})}\left\{\sum_{k=1}^{K} w_{k} h_{k}(\boldsymbol{\theta}, \boldsymbol{t})\right\},
$$

where $w_{k}=n_{k} / n, n=\sum_{k=1}^{K} n_{k}$, and $\mathcal{T}_{n}(\boldsymbol{\theta})=\bigcap_{k=1}^{K} \mathcal{T}_{n_{k}}(\boldsymbol{\theta})$. 
This CEL estimator $\hat{\boldsymbol{\theta}}_{\text {cen }}$ is no difference than the classical EL estimator, so all relevant finite-sample and large-sample properties in the theory of empirical likelihood hold and are applicable for $\hat{\boldsymbol{\theta}}_{\text {cen }}$ under suitable regularity conditions. Arguably, in the case (a) of homogeneity, the CEL method is the method of choice to perform EL estimation and inference as long as a certain adequate computational facility is available, and no data sharing barriers exist across multiple data sources.

\subsection{Doubly constrained DEL method}

As pointed above in Section 1, obtaining the CEL estimator $\hat{\boldsymbol{\theta}}_{c e n}$ in 2.3 can be challenging or even prohibitive in many applications. We propose a solution to address this challenge, called doubly constrained (DOC) DEL (DEL.DOC) method. Inspired by the divide-and-conquer strategy, our solution leads to a new method of distributed estimation and inference in the context of empirical likelihood. Our proposed DEL.DOC method allows to utilize data stored on separate locations without the need of communicating and sharing subject-level information across these individual datasets.

The divide-and-conquer strategy may be implemented by the following 
doubly constrained EL optimization problem:

$$
\min _{\boldsymbol{\Theta} \in \mathcal{D}_{\boldsymbol{\Theta}}} \sup _{\boldsymbol{T} \in \mathcal{D}_{\mathcal{T}_{n}(\boldsymbol{\Theta})}}\left\{\sum_{k=1}^{K} w_{k} h_{k}\left(\boldsymbol{\theta}_{k}, \boldsymbol{t}_{k}\right)\right\},
$$

subject to $\boldsymbol{\theta}_{1}=\boldsymbol{\theta}_{2}=\cdots=\boldsymbol{\theta}_{K}$, and $\boldsymbol{t}_{1}=\boldsymbol{t}_{2}=\cdots=\boldsymbol{t}_{K}$,

where $\boldsymbol{\Theta}=\left(\boldsymbol{\theta}_{1}, \boldsymbol{\theta}_{2}, \cdots, \boldsymbol{\theta}_{K}\right), \boldsymbol{T}=\left(\boldsymbol{t}_{1}, \boldsymbol{t}_{2}, \cdots, \boldsymbol{t}_{K}\right), \mathcal{D}_{\boldsymbol{\Theta}}=\left\{\boldsymbol{\Theta}: \boldsymbol{\Theta}=\left(\boldsymbol{\theta}_{1}, \cdots, \boldsymbol{\theta}_{K}\right)\right.$ with $\left.\boldsymbol{\theta}_{k} \in \boldsymbol{\Xi}\right\}$, and $\mathcal{D}_{\mathcal{T}_{n}(\boldsymbol{\Theta})}=\left\{\boldsymbol{T}: \boldsymbol{T}=\left(\boldsymbol{t}_{1}, \cdots, \boldsymbol{t}_{K}\right)\right.$ with $\left.\boldsymbol{t}_{k} \in \mathcal{T}_{n_{k}}\left(\boldsymbol{\theta}_{k}\right)\right\}$. The double constraints refer to the two sets of equality constraints on $\boldsymbol{\theta}_{k}$ 's and $\boldsymbol{t}_{k}$ 's, respectively, which guarantee the resulting EL estimator equivalent to the centralized EL $\hat{\boldsymbol{\theta}}_{c e n}$ estimation. This optimization problem (2.4) is different from the conventional meta estimation given in (1.1), where the combined estimator is based directly on individual EL $\hat{\boldsymbol{\theta}}_{k}$ 's. In contrast, the proposed DEL.DOC method in (2.4) gives a combined estimator through the aggregated objective function with the equality constraints imposed directly on parameters $\boldsymbol{\theta}_{k}$ 's and $\boldsymbol{t}_{k}$ 's. Such global constraints lead to a new combined EL estimator of $\boldsymbol{\theta}_{0}$. Note that without such constraints, individual parameters $\boldsymbol{\theta}_{k}$ will be estimated separately in parallel with no data integration. To solve (2.4), we invoke the means of alternating direction method of multipliers (ADMM) (Boyd et al. 2011) via the following 
optimization problem:

$$
\begin{aligned}
& \min _{\substack{\boldsymbol{\theta} \in \mathcal{D}_{\boldsymbol{\theta}} \\
\boldsymbol{a} \in \boldsymbol{\Xi}}} \sup _{\substack{\boldsymbol{T} \in \mathcal{D}_{\mathcal{T}_{n}} \\
\boldsymbol{b} \in \mathcal{T}_{n}(\boldsymbol{a})}}\left\{\sum_{k=1}^{K} w_{k} h_{k}\left(\boldsymbol{\theta}_{k}, \boldsymbol{t}_{k}\right)\right\}, \\
& \text { subject to } \boldsymbol{\theta}_{k} \equiv \boldsymbol{a}, \boldsymbol{t}_{k} \equiv \boldsymbol{b}, k=1, \ldots, K .
\end{aligned}
$$

The constrained optimization in 2.5) can be solved via Algorithm 1, whose convergence is established in Proposition 1. The final output estimates are denoted by $\hat{\boldsymbol{\theta}}_{\text {del.doc }}=\boldsymbol{a}^{*}$, namely $\boldsymbol{a}^{*}$ is the converged value of $\boldsymbol{a}^{(s)}$.

Remark 1. In Step 6 of Algorithm 1, updated $\boldsymbol{\theta}_{k}^{(s)}$ is obtained by minimizing a modified empirical likelihood function that is expanded with a quadratic term consisting of the combined estimate $\boldsymbol{a}^{(s)}$, the step size $\boldsymbol{u}_{1, k}^{(s)}$ and the learning rate matrix $\Omega_{1, k}$. The rationale of adding such an expansion in the EL method stems from the fact that this quadratic term in fact pulls separate local estimates towards a common overall estimate, and thus enables to effectively "borrow" information from other datasets during iterations, with no need of accessing subject-level observations of other data sources. In Theorem 1, we show that the estimator $\hat{\boldsymbol{\theta}}_{\text {del.doc }}$ is equal, almost surely, to the CEL $\hat{\boldsymbol{\theta}}_{\text {cen }}$ obtained in (2.3). In other words, the proposed DEL.DOC method can provide the same solution as that obtained by running analyses with the aggregated datasets once. In addition, with this quadratic term, the proposed DEL method overcomes the problem of unbalanced variables, 
as shown in both simulation and real data examples.

In the classical meta-analysis, the meta-estimator in 1.1) takes a simple one-step weighted average of dataset-specific estimators $\hat{\boldsymbol{\theta}}_{k}$ 's via 2.2. With these given estimates $\hat{\boldsymbol{\theta}}_{k}$ 's, proper weights (e.g., estimates of variances $\left.V_{k}\right)$ are chosen to ensure validity of the meta-estimator. In the proposed DEL.DOC method, all separate estimates $\boldsymbol{\theta}_{k}^{(s)}$ are iteratively calibrated by the quadratic term in the ADMM algorithm step (ii), and therefore we can take a relatively liberal choice of learning rate matrix $\Omega_{1, k}$ in the procedure of weighted averaging to combine individual estimates. From this point of view, the meta-estimator 1.1 may be regarded essentially as a one-step approximation to the proposed DEL.DOC estimator. Because DEL.DOC method does not explicitly involve calculation of variances $V_{k}$, it avoids both the difficulty in the estimation of the variance $V_{k}$, and potential numerical instability caused by poor estimate of $V_{k}$ with small sample size $n_{k}$. 


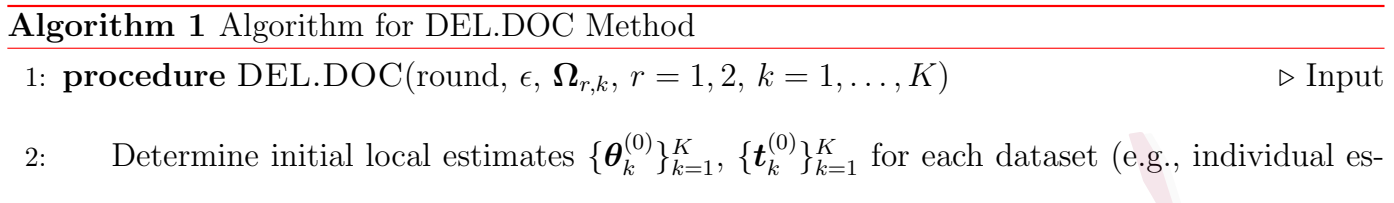
timates $\hat{\boldsymbol{\theta}}_{k}$ and corresponding $\hat{\boldsymbol{t}}_{k}$ ), initial global estimates $\boldsymbol{a}^{(0)}, \boldsymbol{b}^{(0)}$ (e.g., simple averages of local estimates), and initial step sizes $\left\{\boldsymbol{u}_{1 k}^{(0)}\right\}_{k=1}^{K},\left\{\boldsymbol{u}_{2 k}^{(0)}\right\}_{k=1}^{K}$ (e.g., all set at $\mathbf{0}$ ).

3: $\quad$ iter $\leftarrow 1$

4: $\quad$ converge $\leftarrow$ FALSE

5: $\quad$ while !converge \& iter $\leq$ round do

6: $\quad$ Update $\boldsymbol{\theta}_{k}, \boldsymbol{t}_{k}, \boldsymbol{a}, \boldsymbol{b}, \boldsymbol{u}_{1, k}, \boldsymbol{u}_{2, k}$ by

$$
\begin{aligned}
& \boldsymbol{\theta}_{k}^{(s+1)}=\underset{\boldsymbol{\theta}_{k}}{\arg \min }\left\{w_{k} h_{k}\left(\boldsymbol{\theta}_{k}, \boldsymbol{t}_{k}^{(s)}\right)\right. \\
& \left.+\frac{1}{2}\left(\boldsymbol{\theta}_{k}-\boldsymbol{a}^{(s)}+\boldsymbol{u}_{1, k}^{(s)}\right)^{T} \boldsymbol{\Omega}_{1, k}\left(\boldsymbol{\theta}_{k}-\boldsymbol{a}^{(s)}+\boldsymbol{u}_{1, k}^{(s)}\right)\right\} ; \\
& \boldsymbol{t}_{k}^{(s+1)}=\underset{\boldsymbol{t}_{k}}{\arg \min }\left\{-w_{k} h_{k}\left(\boldsymbol{\theta}_{k}^{(s+1)}, \boldsymbol{t}_{k}\right)\right. \\
& \left.+\frac{1}{2}\left(\boldsymbol{t}_{k}-\boldsymbol{b}^{(s)}+\boldsymbol{u}_{2, k}^{(s)}\right)^{T} \boldsymbol{\Omega}_{2, k}\left(\boldsymbol{t}_{k}-\boldsymbol{b}^{(s)}+\boldsymbol{u}_{2, k}^{(s)}\right)\right\} \\
& \boldsymbol{a}^{(s+1)}=\left(\sum_{k=1}^{K} \boldsymbol{\Omega}_{1, k}\right)^{-1}\left(\sum_{k=1}^{K} \boldsymbol{\Omega}_{1, k} \boldsymbol{\theta}_{k}^{(s+1)}\right), \boldsymbol{b}^{(s+1)}=\left(\sum_{k=1}^{K} \boldsymbol{\Omega}_{2, k}\right)^{-1}\left(\sum_{k=1}^{K} \boldsymbol{\Omega}_{2, k} \boldsymbol{t}_{k}^{(s+1)}\right) ; \\
& \boldsymbol{u}_{1, k}^{(s+1)}=\boldsymbol{u}_{1, k}^{(s)}+\boldsymbol{\theta}_{k}^{(s+1)}-\boldsymbol{a}^{(s+1)}, \boldsymbol{u}_{2, k}^{(s+1)}=\boldsymbol{u}_{2, k}^{(s)}+\boldsymbol{t}_{k}^{(s+1)}-\boldsymbol{b}^{(s+1)} ;
\end{aligned}
$$

where $\boldsymbol{\Omega}_{r, k}, r=1,2, k=1, \ldots, K$ are pre-specified $(q+1) \times(q+1)$-dimensional positivedefinite symmetric matrices pertaining to the algorithmic convergence rate.

7: $\quad$ if $\max _{k}\left\|\boldsymbol{\theta}_{k}^{(s+1)}-\boldsymbol{\theta}_{k}^{(s)}\right\|_{2}<\epsilon$ and $\max _{k}\left\|\boldsymbol{t}_{k}^{(s+1)}-\boldsymbol{t}_{k}^{(s)}\right\|_{2}<\epsilon$ for a pre-specified threshold $\epsilon$, say $10^{-4}$ then converge $\leftarrow$ TRUE

8: $\quad$ iter $\leftarrow$ iter +1

9:

return $\Theta, T, a, b$. 


\subsection{Theoretical properties15}

\subsection{Theoretical properties}

In the case of homogeneity, $\boldsymbol{\theta}_{k 0} \equiv \boldsymbol{\theta}_{0}$ and $g_{k}(\cdot) \equiv g(\cdot)$ for all $k$, we let $\boldsymbol{S} \equiv \boldsymbol{S}_{1} \equiv \cdots \equiv \boldsymbol{S}_{K}$ and $\boldsymbol{Q} \equiv \boldsymbol{Q}_{1} \equiv \cdots \equiv \boldsymbol{Q}_{K}$, where $\boldsymbol{S}_{k}$ and $\boldsymbol{Q}_{k}$ are sensitivity and variability matrices defined in conditions (C5) and (C3), respectively. Below we first present the algorithmic convergence of the ADMM algorithm in Proposition 1, which is proposed to implement the DEL.DOC method. Its proof is given in the supplementary materials.

Proposition 1. (Algorithmic convergence of ADMM for DEL.DOC method)

Denote $\boldsymbol{U}_{r}=\left(\boldsymbol{u}_{r, 1}, \cdots, \boldsymbol{u}_{r, K}\right), r=1,2$. Let $L_{01}\left(\boldsymbol{\Theta}, \mathbf{T}, \boldsymbol{a}, \boldsymbol{U}_{1}\right)=\sum_{k=1}^{K} w_{k} h_{k}\left(\boldsymbol{\theta}_{k}, \boldsymbol{t}_{k}\right)+$ $\sum_{k=1}^{K} \boldsymbol{u}_{1, k}^{T} \boldsymbol{\Omega}_{1, k}\left(\boldsymbol{\theta}_{k}-\boldsymbol{a}\right)$, and $L_{02}\left(\boldsymbol{\Theta}, \mathbf{T}, \boldsymbol{b}, \boldsymbol{U}_{2}\right)=-\sum_{k=1}^{K} w_{k} h_{k}\left(\boldsymbol{\theta}_{k}, \boldsymbol{t}_{k}\right)+\sum_{k=1}^{K} \boldsymbol{u}_{2, k}^{T} \boldsymbol{\Omega}_{2, k}\left(\boldsymbol{t}_{k}-\right.$ b). Suppose that (i) there exists $\left(\boldsymbol{\Theta}^{*}, \mathbf{T}^{*}, \boldsymbol{a}^{*}, \boldsymbol{b}^{*}, \boldsymbol{U}_{1}^{*}, \boldsymbol{U}_{2}^{*}\right)$, for which the following inequalities hold for all $\boldsymbol{\Theta} \in \mathcal{D}_{\boldsymbol{\theta}}, \mathbf{T} \in \mathcal{D}_{\mathcal{T}_{n}}, \boldsymbol{a} \in \Xi, \boldsymbol{b} \in \mathcal{T}_{n}(\boldsymbol{a}), \boldsymbol{U}_{1} \in$ $\mathcal{R}^{(q+1) \times K}, \boldsymbol{U}_{2} \in \mathcal{R}^{m_{1}+\cdots+m_{K}}: L_{01}\left(\boldsymbol{\Theta}^{*}, \mathbf{T}, \boldsymbol{a}^{*}, \boldsymbol{U}_{1}\right) \leq L_{01}\left(\boldsymbol{\Theta}^{*}, \mathbf{T}, \boldsymbol{a}^{*}, \boldsymbol{U}_{1}^{*}\right) \leq$ $L_{01}\left(\boldsymbol{\Theta}, \mathbf{T}, \boldsymbol{a}, \boldsymbol{U}_{1}^{*}\right)$, and $L_{02}\left(\boldsymbol{\Theta}, \mathbf{T}^{*}, \boldsymbol{b}^{*}, \boldsymbol{U}_{2}\right) \leq L_{02}\left(\boldsymbol{\Theta}, \mathbf{T}^{*}, \boldsymbol{b}^{*}, \boldsymbol{U}_{2}^{*}\right) \leq L_{02}\left(\boldsymbol{\Theta}, \mathbf{T}, \boldsymbol{b}, \boldsymbol{U}_{2}^{*}\right)$ and (ii) there exist initial values $\left(\boldsymbol{a}^{(0)}, \boldsymbol{b}^{(0)}, \boldsymbol{U}_{1}^{(0)}, \boldsymbol{U}_{2}^{(0)}\right)$ such that

$$
\begin{aligned}
& \sum_{k=1}^{K}\left\{\left(\boldsymbol{u}_{1, k}^{(0)}-\boldsymbol{u}_{1, k}^{*}\right)^{T} \boldsymbol{\Omega}_{1, k}\left(\boldsymbol{u}_{1, k}^{(0)}-\boldsymbol{u}_{1, k}^{*}\right)+\left(\boldsymbol{a}^{(0)}-\boldsymbol{a}^{*}\right)^{T} \boldsymbol{\Omega}_{1, k}\left(\boldsymbol{a}^{(0)}-\boldsymbol{a}^{*}\right)\right\} \leq M_{1}, \\
& \sum_{k=1}^{K}\left\{\left(\boldsymbol{u}_{2, k}^{(0)}-\boldsymbol{u}_{2, k}^{*}\right)^{T} \boldsymbol{\Omega}_{2, k}\left(\boldsymbol{u}_{2, k}^{(0)}-\boldsymbol{u}_{2, k}^{*}\right)+\left(\boldsymbol{b}^{(0)}-\boldsymbol{b}^{*}\right)^{T} \boldsymbol{\Omega}_{2, k}\left(\boldsymbol{b}^{(0)}-\boldsymbol{b}^{*}\right)\right\} \leq M_{2},
\end{aligned}
$$


for some positive constants $M_{1}$ and $M_{2}$. Then, we have as iteration $s \rightarrow \infty$,

$$
\begin{gathered}
\max _{k}\left\|\boldsymbol{\theta}_{k}^{(s)}-\boldsymbol{\theta}_{k}^{*}\right\|_{2} \rightarrow 0, \text { and }\left\|\boldsymbol{a}^{(s)}-\boldsymbol{a}^{*}\right\|_{2} \rightarrow 0 \\
\max _{k}\left\|\boldsymbol{t}_{k}^{(s)}-\boldsymbol{t}_{k}^{*}\right\|_{2} \rightarrow 0, \text { and }\left\|\boldsymbol{b}^{(s)}-\boldsymbol{b}^{*}\right\|_{2} \rightarrow 0
\end{gathered}
$$

Proposition 1 indicates that according to the condition (ii) both initial values and learning rate matrices $\boldsymbol{\Omega}_{r, k}$ could affect the performance of DEL.DOC method. When the number of data sources, $K$, is large, proper initial values are required to ensure convergence of the algorithm. Theorem 1 below gives the almost sure equality between $\hat{\boldsymbol{\theta}}_{\text {del.doc }}$ and $\hat{\boldsymbol{\theta}}_{c e n}$, as well as asymptotic properties of $\hat{\boldsymbol{\theta}}_{\text {del.doc }}$.

Theorem 1. Assume Conditions (C1)-(C5) in the Appendix hold.

(a) If the initial values satisfy $\boldsymbol{a}^{(0)}=\boldsymbol{\theta}_{0}+O\left(K^{-1 / 2}\right), \boldsymbol{b}^{(0)}=O\left(K^{-1 / 2}\right)$, $\boldsymbol{U}_{1}^{(0)}=\mathbf{0}$ and $\boldsymbol{U}_{2}^{(0)}=\mathbf{0}$, then $\operatorname{Prob}\left(\hat{\boldsymbol{\theta}}_{\text {del.doc }}=\hat{\boldsymbol{\theta}}_{\text {cen }}\right)=1$.

(b) If the conditions of initial values given in part (a) hold, as $n \rightarrow \infty$,

$$
\sqrt{n}\left(\begin{array}{c}
\hat{\boldsymbol{\theta}}_{d e l . d o c}-\boldsymbol{\theta}_{0} \\
\hat{\boldsymbol{t}}_{\text {del.doc }}-\mathbf{0}
\end{array}\right) \stackrel{d}{\rightarrow} \mathcal{N}\left(\mathbf{0},\left[\begin{array}{cc}
\boldsymbol{J}_{\text {del.doc }}^{-1} & \mathbf{0} \\
\mathbf{0} & \boldsymbol{Q}^{-1}-\boldsymbol{Q}^{-1} \boldsymbol{S} \boldsymbol{J}_{\text {del.doc }}^{-1} \boldsymbol{S}^{T} \boldsymbol{Q}^{-1}
\end{array}\right]\right)
$$

where the Godambe information matrix $\boldsymbol{J}_{\text {del.doc }}=\boldsymbol{S}^{T} \boldsymbol{Q}^{-1} \boldsymbol{S}$.

The proof of Theorem 1 is given in the Supplementary Materials. According to Theorem 1, when the number of data sources, $K$, is fixed, the 
initial values of $\boldsymbol{a}$ and $\boldsymbol{b}$ may be chosen easily, say, certain reasonable constant vectors. However, if $K$ increases, the initial value of $\boldsymbol{a}^{(0)}$ should be chosen to be close to the true value $\boldsymbol{\theta}_{0}$ at rate $O\left(K^{-1 / 2}\right)$.

\section{DEL method in the partial homogeneity setting}

Now we consider scenario (b) of $\boldsymbol{\theta}_{k 0} \equiv \boldsymbol{\theta}_{0}$ but $g_{k}(\cdot)$ varies over $k$. We propose a singly constrained (SIC) DEL (DEL.SIC) method to perform meta EL estimation. Asymptotic properties of DEL.SIC method are thoroughly investigated in this section.

\subsection{Singly constrained DEL method}

The DEL.DOC method above imposes two sets of equality constraints, $\boldsymbol{\theta}_{k} \equiv \boldsymbol{a}$ and $\boldsymbol{t}_{k} \equiv \boldsymbol{b}$ for $k=1, \ldots, K$, to enforce homogeneity on both parameters $\boldsymbol{\theta}_{k 0}$ and estimation functions $g_{k}(\cdot)$. This is the typical situation considered routinely in the classical meta-analysis. However, it is worth noting that in most of practical studies, the second set of constraints on $g_{k}$ may be rarely satisfied due to inter-data heterogeneity. For example, in the setting of instrumental variable models (Imbens 2002, Newey \& Smith 2004), different datasets may have their own instrumental variables, leading to different numbers of moment constraints. Thus, $g_{k}(\cdot)$ may vary not 


\subsection{Singly constrained DEL method18}

only in their forms but in their dimensions. In this case, $\boldsymbol{t}_{k}$ are of different dimensions; hence it is impossible to require $\boldsymbol{t}_{k} \equiv \boldsymbol{b}$. A natural modification of the previous DEL.DOC method is to remove the second set of constraints on $\boldsymbol{t}_{k}$, resulting in a relaxed DEL method with only a single set of constraints $\boldsymbol{\theta}_{k} \equiv \boldsymbol{a}$. Interestingly, as shown in Theorem 3, this generalization is advantageous to handle the homogeneity case (a), in which the resulting DEL.SIC estimator appears to have smaller asymptotic variances than those obtained by the doubly constrained DEL.DOC method.

Specifically, DEL.SIC method relaxes step 6 in Algorithm 1, given as follows:

$$
\begin{aligned}
& \boldsymbol{\theta}_{k}^{(s+1)}=\underset{\boldsymbol{\theta}_{k}}{\arg \min }\left\{w_{k} f_{k}\left(\boldsymbol{\theta}_{k}\right)+\frac{1}{2}\left(\boldsymbol{\theta}_{k}-\boldsymbol{a}^{(s)}+\boldsymbol{u}_{k}^{(s)}\right)^{T} \boldsymbol{\Omega}_{k}\left(\boldsymbol{\theta}_{k}-\boldsymbol{a}^{(s)}+\boldsymbol{u}_{k}^{(s)}\right)\right\} ; \\
& \boldsymbol{a}^{(s+1)}=\left(\sum_{k=1}^{K} \boldsymbol{\Omega}_{k}\right)^{-1}\left(\sum_{k=1}^{K} \boldsymbol{\Omega}_{k} \boldsymbol{\theta}_{k}^{(s+1)}\right) ; \boldsymbol{u}_{k}^{(s+1)}=\boldsymbol{u}_{k}^{(s)}+\boldsymbol{\theta}_{k}^{(s+1)}-\boldsymbol{a}^{(s+1)} ; \quad(3.6)
\end{aligned}
$$

where $f_{k}(\boldsymbol{\theta})=\sup _{\boldsymbol{t} \in \mathcal{T}_{n_{k}}(\boldsymbol{\theta})}\left\{n_{k}^{-1} \sum_{i=1}^{n_{k}} \log \left(1-\boldsymbol{t}^{T} g_{k i}(\boldsymbol{\theta})\right)\right\}$, and $\boldsymbol{\Omega}_{k}$ is a prespecified learning rate. Denote the converged value of $\boldsymbol{a}^{(s)}$ from the relaxed ADMM algorithm above as $\hat{\boldsymbol{\theta}}_{\text {del.sic }}$. The convergence of the ADMM algorithm implemented for DEL.SIC method via (3.6), stated in Proposition 2 in the supplementary materials, can also be proved by similar arguments as those in Proposition 1. See the detail in the supplementary materials.

Remark 2. DEL.SIC method provides an interesting interpretation as a 


\section{$3.2 \quad$ Asymptotic properties19}

new approach to aggregating empirical likelihoods. Consider an objective function $L(\boldsymbol{\theta})$ that aggregates $K$ individual empirical likelihoods, each having its own set of $p_{k i}{ }^{\prime} s: L(\boldsymbol{\theta})=\sup _{p_{k i}} \prod_{k=1}^{K} \prod_{i=1}^{n_{k}} n_{k} p_{k i}$, subject to $p_{k i} \geq$ $0, \sum_{i=1}^{n_{k}} p_{k i}=1, \quad \sum_{i=1}^{n_{k}} p_{k i} g_{k i}(\boldsymbol{\theta})=0, k=1, \ldots, K$. In this formulation, different $g_{k}(\cdot)$ are allowed in the aggregation. Let $\check{\boldsymbol{\theta}}_{\text {cen }}$ be the EL solution to the above objective function obtained by a centralized computation method. After some simple calculations, it is easy to obtain that

$$
\check{\boldsymbol{\theta}}_{c e n}=\underset{\boldsymbol{\theta} \in \Xi}{\arg \min } \sum_{k=1}^{K} w_{k} f_{k}(\boldsymbol{\theta})=\underset{\boldsymbol{\theta} \in \Xi}{\arg \min }\left\{\sum_{k=1}^{K} w_{k} \sup _{\boldsymbol{t}_{k} \in \mathcal{T}_{n_{k}}(\boldsymbol{\theta})} h_{k}\left(\boldsymbol{\theta}, \boldsymbol{t}_{k}\right)\right\} .
$$

The relaxed ADMM algorithm (3.6) enables us to search for the solution to a reformulated optimality problem given in (3.7) with the following constraints: $\min _{\boldsymbol{\Theta} \in \mathcal{D}_{\boldsymbol{\Theta}}} \sup _{\boldsymbol{T} \in \mathcal{D}_{\mathcal{T}_{n}(\boldsymbol{\Theta})}}\left\{\sum_{k=1}^{K} w_{k} h_{k}\left(\boldsymbol{\theta}_{k}, \boldsymbol{t}_{k}\right)\right\}$, subject to $\boldsymbol{\theta}_{1}=\cdots=\boldsymbol{\theta}_{K}$. It indeeds coincides with the proposed estimator $\hat{\boldsymbol{\theta}}_{\text {del.sic }}$. The next subsection presents asymptotic properties of the CLE $\check{\boldsymbol{\theta}}_{c e n}$, and present the almost sure equality between $\check{\boldsymbol{\theta}}_{\text {cen }}$ and $\hat{\boldsymbol{\theta}}_{\text {del.sic }}$.

\subsection{Asymptotic properties}

Asymptotic consistency and normality of $\check{\boldsymbol{\theta}}_{\text {cen }}$ are established in Theorems 2 and 3 , both of which are new due to the relaxation with varying $g_{k}(\cdot)$ in the EL method. Theorem 5 presents the almost sure equality between $\check{\boldsymbol{\theta}}_{\text {cen }}$ and $\hat{\boldsymbol{\theta}}_{\text {del.sic }}$. Theorem 6 concerns asymptotic distribution of the empirical 
likelihood ratio statistic used for a distributed inference. All the proofs are given in the supplementary materials. Let $n_{\min } \stackrel{\text { def }}{=} \min \left\{n_{1}, \cdots, n_{K}\right\}$.

Theorem 2. (Consistency of $\check{\boldsymbol{\theta}}_{\text {cen }}$ ) If Conditions (C1)-(C3) in the Appendix hold, then $\check{\boldsymbol{\theta}}_{\text {cen }} \stackrel{p}{\rightarrow} \boldsymbol{\theta}_{0}$ as $n_{\text {min }} \rightarrow \infty$. Moreover, let $\check{g}_{k}=n_{k}^{-1} \sum_{i=1}^{n_{k}} g_{k}\left(\boldsymbol{W}_{k i} ; \check{\boldsymbol{\theta}}_{\text {cen }}\right)$ and $\check{\boldsymbol{t}}_{k}=\arg \max _{\boldsymbol{t} \in \mathcal{T}_{n_{k}}\left(\check{\boldsymbol{\theta}}_{c e n}\right)} w_{k} h_{k}\left(\check{\boldsymbol{\theta}}_{c e n}, \boldsymbol{t}\right)$, We have (i) $\check{g}_{k}=O_{p}\left(n_{k}^{-1 / 2}\right)$; (ii) $\check{\boldsymbol{t}}_{k}$ exists with probability approaching one; and (iii) $\check{\boldsymbol{t}}_{k}=O_{p}\left(n_{k}^{-1 / 2}\right)$.

The estimation consistency above is well known in the literature.

Theorem 3. (Asymptotic normality of $\check{\boldsymbol{\theta}}_{\text {cen }}$ ) Under Conditions (C1)-(C5) in the Appendix, if $K=O\left(n^{1 / 2-\delta}\right)$ for some $0<\delta \leq 1 / 2$ and $n_{\min } \rightarrow \infty$, we have $\left(\begin{array}{c}\sqrt{n}\left(\check{\boldsymbol{\theta}}_{c e n}-\boldsymbol{\theta}_{0}\right) \\ \sqrt{n_{1}}\left(\check{\boldsymbol{t}}_{1}-\mathbf{0}\right) \\ \vdots \\ \sqrt{n_{K}}\left(\check{\boldsymbol{t}}_{K}-\mathbf{0}\right)\end{array}\right) \stackrel{d}{\rightarrow} \mathcal{N}\left(\mathbf{0},\left[\begin{array}{cccc}\boldsymbol{J}_{\text {del.sic }}^{-1} & \mathbf{0} & \cdots & \mathbf{0} \\ \mathbf{0} & \boldsymbol{Q}_{1}^{-1}-w_{1} \boldsymbol{P}_{11} & \cdots & -w_{1} \boldsymbol{P}_{1 K} \\ \vdots & \vdots & \cdots & \vdots \\ \mathbf{0} & -w_{K} \boldsymbol{P}_{K 1} & \cdots & \boldsymbol{Q}_{K}^{-1}-w_{K} \boldsymbol{P}_{K K}\end{array}\right]\right)$, where $\boldsymbol{J}_{\text {del.sic }}=\lim _{n_{\min } \rightarrow \infty}\left(\sum_{k=1}^{K} w_{k} \boldsymbol{S}_{k}^{T} \boldsymbol{Q}_{k}^{-1} \boldsymbol{S}_{k}\right), \boldsymbol{P}_{i j}=\boldsymbol{Q}_{i}^{-1} \boldsymbol{S}_{i} \boldsymbol{J}_{\text {del.sic }}^{-1} \boldsymbol{S}_{j}^{T} \boldsymbol{Q}_{j}^{-1}$.

The next Theorem presents a comparison of estimation efficiency between $\check{\boldsymbol{\theta}}_{c e n}$ in $(3.7)$ and $\hat{\boldsymbol{\theta}}_{c e n}$ in $(2.3)$.

Theorem 4. If the dimensions of $g_{k}(\cdot)$ are the same, i.e., $m_{1}=\cdots=m_{K}$, then $\boldsymbol{J}_{\text {del.sic }} \geq \boldsymbol{J}_{\text {cen }}$, where $\boldsymbol{J}_{\text {cen }}=\lim _{n \rightarrow \infty}\left\{\left(\sum_{k=1}^{K} w_{k} \boldsymbol{S}_{k}^{T}\right)\left(\sum_{k=1}^{K} w_{k} \boldsymbol{Q}_{k}\right)^{-1}\left(\sum_{k=1}^{K} w_{k} \boldsymbol{S}_{k}\right)\right\}$ 
is the asymptotic covariance of the centralized EL estimator $\hat{\boldsymbol{\theta}}_{\text {cen }}$ in 2.3.). The inequality $\geq$ is in the Löwner's sense. Moreover, the equal variance occurs if and only if $\boldsymbol{S}_{k}^{T} \equiv \boldsymbol{Q}_{k}, k=1, \ldots, K$, or $\boldsymbol{S}_{1}^{T} \boldsymbol{Q}_{1}^{-1}=\cdots=\boldsymbol{S}_{K}^{T} \boldsymbol{Q}_{K}^{-1}$.

Theorem 4 implies an interesting result given as follows.

Corollary 1. In the homogeneity case (a), $\hat{\boldsymbol{\theta}}_{\text {cen }}$ (equialent to $\hat{\boldsymbol{\theta}}_{\text {del.doc }}$ a.s.) and $\check{\boldsymbol{\theta}}_{\text {cen }}$ have the same estimation efficiency.

Theorem 5. Under the same conditions of Theorem 3, if the initial values $\boldsymbol{a}^{(0)}=\boldsymbol{\theta}_{0}+O\left(K^{-1 / 2}\right)$ and $\boldsymbol{u}^{(0)}=\mathbf{0}$, then $\operatorname{Prob}\left(\hat{\boldsymbol{\theta}}_{\text {del.sic }}=\check{\boldsymbol{\theta}}_{\text {cen }}\right)=1$.

The proof of Theorem 5 is similar to that of Theorem 1 by taking $\left(\boldsymbol{\Theta}^{*}, \boldsymbol{a}^{*}, \boldsymbol{u}^{*}\right)=\left(\check{\boldsymbol{\Theta}}_{c e n}, \check{\boldsymbol{\theta}}_{c e n}, \mathbf{0}\right)$, and thus the detail is omitted here.

Remark 3. Under Theorem 5, it is easy to see Theorems 2- 4 also hold

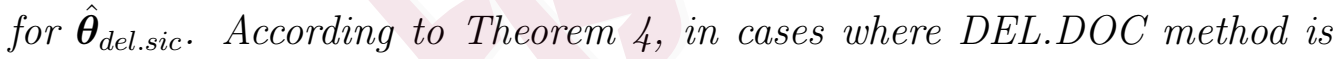
applicable, the asymptotic variance of $\hat{\boldsymbol{\theta}}_{\text {del.sic }}\left(\right.$ or $\left.\check{\boldsymbol{\theta}}_{\text {cen }}\right)$ is no larger than the variance of $\hat{\boldsymbol{\theta}}_{\text {del.doc }}\left(\right.$ or $\left.\hat{\boldsymbol{\theta}}_{\text {cen }}\right)$.

Theorem 3 indicates that the asymptotic normality of $\hat{\boldsymbol{\theta}}_{\text {del.sic }}$ holds when the number of datasets $K$ may grow at a slower rate than $\sqrt{n}$, and all sample sizes of the datasets should tend to infinity. This order of $K, O\left(n^{1 / 2-\delta}\right)$, in Theorem 3 may be relaxed by invoking a high-order bias correction technique. This is because aggregating EL estimates across $K$ datasets reduces 
$3.2 \quad$ Asymptotic properties22

the order of the variance but does not reduce the order of the estimation bias. Denote $\boldsymbol{\Upsilon}=\left(\boldsymbol{\theta}^{T}, \boldsymbol{t}_{1}^{T}, \cdots, \boldsymbol{t}_{K}^{T}\right)^{T}$. Following Firth (1993), we consider a $q$-th order Taylor expansion of $Q(\Upsilon)$, the estimating function to solve the optimization given in (3.7), from which we construct a high-order bias corrected estimator: $\tilde{\boldsymbol{\Upsilon}}_{q}=\check{\Upsilon}+S^{-1} \boldsymbol{r}_{q}$, where $\check{\Upsilon}=\left(\check{\boldsymbol{\theta}}_{\text {cen }}^{T}, \check{\boldsymbol{t}}_{1}^{T}, \cdots, \check{\boldsymbol{t}}_{K}^{T}\right)^{T}, S$ is a $p \times p$ matrix $E\left[\nabla Q\left(\mathbf{\Upsilon}_{0}\right)\right]$ and $\boldsymbol{r}_{q}$ is a certain $p$-element vector yielded from the $q$-order Taylor expansion. We can show that the order of the estimation bias for this new estimator $\tilde{\Upsilon}_{q}$ is $K^{q+2} n^{-(q+1)}+(K / n)^{(q+1) / 2}$. In order for such bias to be asymptotically ignorable, it is sufficient to set $K=o\left(n^{1-\frac{1.5}{q+2}}\right)$ for $q \geq 1$ at which, as shown, the resulting bias is at a higher order than $n^{-1 / 2}$. The technique details can be found in the supplementary materials due to limited space.

Additionally, Theorem 3 implies that (a) In DEL.SIC method, $\hat{\boldsymbol{\theta}}_{\text {del.sic }}$ and $\hat{\boldsymbol{t}}_{k}$ 's are asymptotically independent; (b) $\hat{\boldsymbol{\theta}}_{\text {del.sic }}\left(\right.$ or $\left.\check{\boldsymbol{\theta}}_{c e n}\right)$ has the same convergence rate as that of $\hat{\boldsymbol{\theta}}_{\text {del.doc }}$ and $\hat{\boldsymbol{\theta}}_{c e n} ;$ (c) from Theorem 1 , the asymptotic variance of the individual estimator for $\boldsymbol{t}$ based only on the $k^{\text {th }}$ dataset is $n_{k}^{-1}\left[\boldsymbol{Q}_{k}^{-1}-\boldsymbol{Q}_{k}^{-1} \boldsymbol{S}_{k}\left(\boldsymbol{S}_{k}^{T} \boldsymbol{Q}_{k}^{-1} \boldsymbol{S}_{k}\right)^{-1} \boldsymbol{S}_{k}^{T} \boldsymbol{Q}_{k}^{-1}\right]$, which is different from that of $\hat{\boldsymbol{t}}_{k}$ in DEL.SIC method. Here $\hat{\boldsymbol{t}}_{k}=\arg \max _{\boldsymbol{t} \in \mathcal{T}_{n_{k}}\left(\hat{\boldsymbol{\theta}}_{\text {del.sic }}\right)} w_{k} h_{k}\left(\hat{\boldsymbol{\theta}}_{\text {del.sic }}, \boldsymbol{t}\right)$.

Following the EL literature, we consider an empirical likelihood ratio (ELR) statistic for testing $H_{0}: \boldsymbol{\theta}=\boldsymbol{\theta}_{0}$ as follows: $W_{E}\left(\boldsymbol{\theta}_{0}\right)=2 n\left\{\mathcal{L}\left(\hat{\boldsymbol{\theta}}_{\text {del.sic }}, \hat{\mathbf{T}}\right)-\mathcal{L}\left(\boldsymbol{\theta}_{0}, \mathbf{T}_{0}\right)\right\}$, 
where $\boldsymbol{t}_{k 0}=\arg \max _{\boldsymbol{t} \in \mathcal{T}_{n_{k}}\left(\boldsymbol{\theta}_{0}\right)} h_{k}\left(\boldsymbol{\theta}_{0}, \boldsymbol{t}\right)$ for $k=1, \cdots, K$, and $\mathcal{L}(\boldsymbol{\theta}, \mathbf{T})=$ $\sum_{k=1}^{K} w_{k} h_{k}\left(\boldsymbol{\theta}, \boldsymbol{t}_{k}\right)$ with $\mathbf{T}_{0}=\left(\boldsymbol{t}_{10}, \boldsymbol{t}_{20}, \cdots, \boldsymbol{t}_{K 0}\right)$.

Theorem 6. Under the conditions of Theorem 3 and under the null hypothesis $H_{0}: \boldsymbol{\theta}=\boldsymbol{\theta}_{0}, W_{E}\left(\boldsymbol{\theta}_{0}\right) \stackrel{d}{\rightarrow} \chi_{q+1}^{2}$ as $n_{\min } \rightarrow \infty$ and $\sum_{k=1}^{K} n_{k}^{-1 / 2} \rightarrow 0$.

Note that when all datasets are of equal size, i.e., $n_{k} \equiv \tilde{n}$, Theorem 6 requires that $\tilde{n} \rightarrow \infty$ and $K=O\left(\tilde{n}^{1 / 3-\delta}\right)$ for some $0<\delta \leq 1 / 3$. To construct a confidence interval, we consider the profile empirical likelihood ratio statistic proposed in Qin \& Lawless 1994. Corollary 5). Let $\boldsymbol{\theta}^{T}=\left(\theta_{1}, \boldsymbol{\theta}_{2}^{T}\right)^{T}$, where $\theta_{1}$ is the parameter of interest and $\boldsymbol{\theta}_{2}$ is the subvector of the nuisance parameters, respectively. For $H_{0}: \theta_{1}=\theta_{10}$, the profile empirical likelihood ratio test statistic is $W_{2}=2 n\left(\mathcal{L}\left(\hat{\boldsymbol{\theta}}_{\text {del.sic }}, \hat{\boldsymbol{T}}\right)-\mathcal{L}\left(\theta_{10}, \hat{\boldsymbol{\theta}}_{2}^{\text {null }}, \hat{\boldsymbol{T}}^{\text {null }}\right)\right)$, where $\left(\hat{\boldsymbol{\theta}}_{2}^{\text {null }}, \hat{\boldsymbol{T}}^{\text {null }}\right)=\arg \min _{\boldsymbol{\theta}_{2}} \sup _{\boldsymbol{T}}\left\{\sum_{k=1}^{K} w_{k} h_{k}\left(\boldsymbol{\theta}_{k}, \boldsymbol{t}_{k}\right)\right\}$, subject to $\theta_{1 k} \equiv \theta_{10}, \boldsymbol{\theta}_{2 k} \equiv$ $\boldsymbol{\theta}_{2}, k=1, \cdots, K$, where $\boldsymbol{\theta}_{2}$ is a common subvector across $K$ datasets to be estimated. Following Theorem $6, W_{2} \rightarrow \chi_{1}^{2}$, as $n_{\text {min }} \rightarrow \infty$. The technique details can be found in the supplementary materials. Thus, a $(1-\alpha) 100 \%$ confidence interval satisfies $\operatorname{Prob}\left[W_{2} \leq \chi_{1}^{2}(1-\alpha)\right]=1-\alpha$. We numerically find the lower and upper limits of a $95 \%$ confidence interval, $\left[\hat{\theta}_{1}^{l}, \hat{\theta}_{1}^{u}\right]$, by the following steps: (i) calculate the 0.05 upper quantile of $\chi_{1}^{2}$, denoted by $q_{0.05}$ such that $F\left(q_{0.05}\right)=0.95$, where $F$ is the chi-square distribution function with degree 1 ; (ii) solving the equation $W_{2}\left(\theta_{1}\right)=q_{0.05}$ gives two 
roots $\hat{\theta}_{1}^{u}>\hat{\theta}_{1}^{l}$. Then, the interval length is calculated by $\left|\hat{\theta}_{1}^{u}-\hat{\theta}_{1}^{l}\right|$.

\section{Examples and Numerical Illustration}

Example 1 (Estimation with auxiliary information) In this example, we revisit a two-sample problem considered in Qin \& Lawless (1994) from the perspective of distributed EL estimation and inference. We simulate i.i.d. observations of trivariate random variables $(X, Y, Z)$ according to the following models: $X \sim \operatorname{Weibull}(2,1), Y=X+\epsilon_{1}, Z=X+\epsilon_{2}$, where $\left(\epsilon_{1}, \epsilon_{2}\right) \sim \operatorname{BVN}(0,0,1,1,-0.6)$ and $\left(\epsilon_{1}, \epsilon_{2}\right)$ are independent of $X$. We consider a scenario of $K=3$ datasets with unequal sample sizes. The parameter of interest is a tail probability $p=P\left(X \geq \xi_{0.9}\right)$, with $\xi_{0.9}$ being the 0.9 -quantile of Weibull $(2,1)$. Clearly, the true value $p_{0}=0.1$. Suppose that there is additional information from certain external sources $(Y, Z)$ of, say, $E(Y)=\mu_{y 0}=2$ and $E(X)=E(Z)$. Such auxiliary information is relevant to parameter $p$ since $X$ is correlated with two auxiliary variables $(Y, Z)$. To incorporate this information in the estimation of $p$, we may set up a joint estimating function of the following form: $g(x, y, z ; p)=\left(\mathbf{1}\left(x \geq \xi_{0.9}\right)-p, y-\mu_{y 0}, x-z\right)^{T}$, which is unbiased because $E\left\{g\left(X, Y, Z ; p_{0}\right)\right\}=\mathbf{0}$. We assess the performance of DEL.DOC method with comparison to the following methods: (i) the naive sample propor- 
tion estimator with no use of the auxiliary information, (ii) the centralized EL (CEL) estimator based on the combined dataset, and (iii) the classical meta estimator via the inverse variance weighting. For the meta estimator, individual variances $V_{k}$ are separately estimated using the EL asymptotic variance given in Qin \& Lawless (1994). Table 1 shows the summary results over 2000 replicates under different sample sizes. The results include average bias, empirical standard error (ESE), average asymptotic standard error (ASE), coverage probability (CP), average interval length (AIL) of 95\% confidence interval and average computational time (Time) measured in seconds. ASEs are reported only for both naive and meta estimators based on the asymptotic normality. The two methods use the estimated asymptotic variances in the construction of confidence intervals, while all the other methods utilize the empirical likelihood ratio statistic according to Theorem 6. Table 1 shows that the performance of the proposed DEL.DOC method is virtually identical to that of the centralized EL method, where their minor differences are due mostly to numerical implementation. These two top performers give very small estimation biases and adequate coverage probabilities under all cases of sample sizes considered. The meta method clearly underestimates the standard error with a noticeably lower coverage probability than $95 \%$, especially when some of the datasets have 
small sample sizes. The coverage probability of DEL.SIC method is lower than the nominal $95 \%$ level with the small sample size for $n=60,120$ and $n_{k}=20,40$. This is not surprising as DEL.SIC method requires individual $n_{k} \rightarrow \infty$. The meta method performs the worst with much lower coverage probabilities around $70 \%$ and $80 \%$. When all individual datasets are sufficiently large, the results of the meta estimation are close to those of the two top methods. Also, the naive estimation method has the largest ESE and AIL because it does not use the auxiliary information in the estimation.

Example 2 (Log-linear model with over-dispersion) This example concerns violation of homogeneity in the sense that the second-order moments of $g_{k}(\cdot)$ are different across datasets due to heterogeneous overdispersion. We simulate i.i.d. observations of $(Y, X)$ from the following Poisson-gamma model: $Y \mid \theta, X \sim \operatorname{Poi}(\theta \mu)$ with $\log (\mu)=\beta_{0}+\beta_{1} X, X \sim$ Uniform $[-1,1]$, where $\left(\beta_{0}, \beta_{1}\right)=(0,1)$ and $\theta$ is a multiplicative random effect with $E(\theta)=1$, whose distribution will be specified differently over $K=3$ datasets. Specifically, for the 1 st dataset, $\theta$ is degenerated as of $\theta \equiv 1$, so no over-dispersion exists; for the 2nd dataset, $\theta$ follows a gamma distribution, $\theta \sim \operatorname{Gamma}(5,5)$; and for the 3rd dataset, $\theta$ follows a two-point distribution, $\theta \in\{0,4\}$ with $P(\theta=4)=1 / 4$. With the correctly-specified marginal mean model, the goal is to estimate the slope parameter $\beta_{1}$. Unbiased esti- 
mating function is given by $g(x, y ; \boldsymbol{\beta})=(1, x)^{T}\left\{y-\exp \left(\beta_{0}+\beta_{1} x\right)\right\}$, which satisfies $E[g(Y, X ; \boldsymbol{\beta})]=\mathbf{0}$. Despite the absence of random effects in the estimating function, here the outcome $y$ is over-dispersed with different dispersion parameters over three datasets. To evaluate the influence of heterogeneity in variances, we apply both DEL.DOC and DEL.SIC methods to estimate $\beta_{1}$. Their performances are also compared with the standard GLM method under no over-dispersion, the centralized EL method based on the combined dataset and the meta method with inverse variance weighting. Results are summarized from 2,000 replicates in Table 2, where the last column lists the relative $95 \%$ confidence interval length (RIL) of a method in comparison to that of the centralized EL (CEL) method.

Similar to the findings in Example 1, the DEL.DOC method and the CEL method exhibit again almost identical performances. On the other hand, the DEL.SIC method shows very close results to the meta EL method, both having smaller ESEs and shorter AILs than the DEL.DOC and the CEL methods. Such numerical evidence confirms the theoretical results in Theorem 3 in a case of heterogeneity, when the second-order moments of estimating functions $g_{k}$ are different due to varying over-dispersion generated by different distributions of random effects. When naively using the standard GLM method with the over-dispersion ignored, although produc- 
ing consistent point estimates, it severely underestimates the variance of the estimator. One message learned from this example is that DEL.SIC method automatically adjusted for heterogeneous over-dispersion without explicitly modeling on it, which is rather appealing in practical studies.

Example 3 (Log-linear model with unbalanced variables) This example concerns data with unbalanced variables. We simulate i.i.d. observations of $\left(Y, X_{1}, X_{2}, X_{3}\right)$ from the following Poisson regression model: $Y \mid X \sim$ $\operatorname{Po}(\mu)$ with $\log (\mu)=\beta_{0}+\beta_{1} X_{1}+\beta_{2} X_{2}+\beta_{3} X_{3}$, where $\left(\beta_{0}, \beta_{1}, \beta_{2}, \beta_{3}\right)=$ $(0,1,1,1)$, and $X_{1} \sim \mathrm{U}[-1,1]$. To create imbalance, $X_{i 2}$ and $X_{i 3}$ are independently generated from Bernoulli $B(1, p)$ with varying $p$. Set the total sample size of $n=300$ subjects that are randomly assigned to $K=10$ datasets satisfying $n_{k} \equiv 30$. Five different levels of unbalanced variables are considered with $p=0.05,0.1,0.2,0.5$. To mimic the real kidney transplant data, we further consider an extremely unbalanced situation (S0) with $X_{2} \sim B(1,0.05)$, while one dataset is generated by $X_{3} \sim B(1,0.5)$, one dataset has $X_{3} \equiv 0$, and the other 8 datasets have $X_{3} \equiv 1$. We compare the proposed DEL method with the centralized GLM method, centralized EL method, the meta GLM method, and the meta EL method. BIAS, ESE, CP and AIL of 95\% confidence interval, proportion of algorithmic convergence (PAC), and computation time (Time) from 2000 replicates are 
reported in Table 3. It is easy to see that DEL method has exactly the same performance as the CEL method regardless of imbalance levels. However, these two meta-type methods fail as imbalance becomes noticeably severe.

\section{Real data example}

The Scientific Registry of Transplant Recipients (SRTR) provides epidemiological data and statistical analysis related to solid organ transplantation in the USA. Post-transplantation graft survival is the clinical outcome of most importance for patients who receive kidney transplant, and understanding

its associated risk factors is of clinical interest. Graft failure at the $5^{\text {th }}$ year ( 1 for yes and 0 for no) after organ replacement therapy is analyzed using a logistic model that includes the following 8 covariates: donor's and recipient's age, BMI (0 if no obese and 1 if obese) and gender ( 0 for female and 1 for male), as well as recipient's previous transplant (prev_tx, 0 for no and 1 for yes) and hepatitis C serology (ree_hcv, 0 for negative and 1 for positive). This illustrative analysis concerns kidney transplant recipients from 3 regions: Alaska (AK), Guam (GU), Wyoming (WY), that has data size 449, 9, and 401, respectively, during the period of 1987 to 2017. Due to limited data size in GU, covariates don_bmi, prev_tx and rec_hcv take the same values with no variability and thus cannot be included in the regression 
model as independent variables. Because the standard logistic regression is not feasible for the data in region GU, the meta method fails to combine results from these 3 regions. For the purpose of comparison, we apply the method only for two regions, AK and WY; see the results in Table 4 under Meta2, in addition to the region-specific logistic regression analyses on AK (GLM_AK) and on WY (GLM_WY) using the R package GLM based on the maximum likelihood estimation. In contrast, our proposed DEL method still works for combining the three regions, and generates several interesting results; see Table 4. From the DEL analysis of SRTR data from the three regions, we see that (i) male donors give higher 5-year graft survival than female donors, whereas male recipients have lower 5-year graft survival than female recipients; (ii) older recipients tend to have a slightly lower 5-year graft failure risk than younger recipients; (iii) recipients who receive repeated transplants tend to have the same 5-year graft survival in comparison to those taking the first-time transplant.

\section{Discussion}

This paper develops a distributed empirical likelihood (DEL) methodology to perform EL estimation and inference with no need of pooling individual datasets together nor sharing subject-level information across multiple 
datasets. It presents a useful approach to protecting data privacy and overcoming data sharing barriers in practice. Two forms of the DEL method is proposed, namely the DEL.SIC and DEL.DOC methods, under the homogeneity and heterogeneity scenarios, respectively. The former is the setting routinely postulated in the classical meta-analysis. Both analytic and numerical results in this paper show that the DEL method is almost surely equivalent to the centralized EL method that processes the aggregated data at a centralized operation platform.

We notice an interesting connection between the meta-estimation and the DEL method. That is, the one-step update of the DEL.SIC method in equation (3.6) indeed gives rise to a meta-estimate 1.1. When the weighting matrix is specified by the variance of the estimator, the resulting meta-estimator is equivalent in distribution to the centralized EL estimator. In contrast, our DEL estimator implemented with the ADMM-based iterative weighting is almost surely equal to the centralized EL estimator. It is known that the mode of almost sure equivalency is stronger than the mode of equivalency in distribution. One key practical advantage of the DEL method is its ability of handling unbalanced data distributions across multiple datasets, a case that often occurs in discrete variables. The metaestimation method may fail when the imbalance appears extreme, although 
such method is computationally faster than the DEL method.

In practice, data heterogeneity appears in various forms, which calls effectively for context-dependent solutions. Our contributions to the DEL method may provide some useful techniques to study data heterogeneity across multiple data sources. Two possible directions of future research include sparsity regularization of parameter $\boldsymbol{\theta}$ and post-fusion inference. The former pertains to the issue of reconciling sparse solutions obtained from different datasets, while the latter involves a certain debiasing procedure to correct estimation bias for valid statistical inferences.

\section{Appendix: Conditions}

Here are regularity conditions required to establish key theoretical properties for the proposed DEL.DOC estimators.

(C1) The true parameter $\boldsymbol{\theta}_{0}$ is an interior point in a compact set $\boldsymbol{\Xi}$ and is the unique solution to $E_{\boldsymbol{\theta}_{0}}\left\{g_{k}\left(\boldsymbol{W}_{k i} ; \boldsymbol{\theta}\right)\right\}=\mathbf{0}, i=1, \ldots, n_{k}, k=$ $1, \ldots, K$

(C2) Estimating function $g_{k}\left(\boldsymbol{W}_{k i} ; \boldsymbol{\theta}\right)$ is continuous at each $\boldsymbol{\theta} \in \boldsymbol{\Xi}$ with probability 1 for $i=1, \ldots, n_{k}, k=1, \ldots, K$, and for some $\alpha>2$, $E_{\boldsymbol{\theta}_{0}}\left(\sup _{\boldsymbol{\theta} \in \boldsymbol{\Xi}, 1 \leq k \leq K}\left\|g_{k}\left(\boldsymbol{W}_{k i} ; \boldsymbol{\theta}\right)\right\|_{2}^{\alpha}\right)<\infty$. 
(C3) Variability matrices $\boldsymbol{Q}_{k}=E_{\boldsymbol{\theta}_{0}}\left\{g_{k}\left(\boldsymbol{W}_{k i} ; \boldsymbol{\theta}_{0}\right) g_{k}^{T}\left(\boldsymbol{W}_{k i} ; \boldsymbol{\theta}_{0}\right)\right\}, k=1, \ldots, K$, are positive-definite.

(C4) $g_{k}\left(\boldsymbol{W}_{k i} ; \boldsymbol{\theta}\right)$ is continuously differentiable in a neighborhood of $\boldsymbol{\theta}_{0}$, say $\mathcal{D}$, and $E_{\boldsymbol{\theta}_{0}}\left(\sup _{\boldsymbol{\theta} \in \mathcal{D}, 1 \leq k \leq K}\left\|\partial g_{k}\left(\boldsymbol{W}_{k i} ; \boldsymbol{\theta}\right) / \partial \boldsymbol{\theta}^{T}\right\|_{2}\right)<\infty$.

(C5) $\operatorname{rank}\left(\boldsymbol{S}_{k}\right)=q+1$, where sensitivity matrix $\boldsymbol{S}_{k}=E_{\boldsymbol{\theta}_{0}}\left\{\partial g_{k}\left(\boldsymbol{W}_{k i} ; \boldsymbol{\theta}_{0}\right) / \partial \boldsymbol{\theta}\right\}$.

All these five conditions (C1)-(C5) are assumed in the seminal work of empirical likelihood (EL) by Qin \& Lawless (1994) in the context of estimating functions. Condition (C1) is a mild regularity condition that requires unbiased estimating functions for estimation consistency, while conditions (C3) and (C5) are routinely imposed in order to obtain a valid sandwich covariance matrix in the asymptotic normality. Conditions (C2) and (C4) appear slightly stronger with the uniform upper bounds than the classical situation of one dataset, which is postulated to deal with the case of $K \rightarrow \infty$. In other words, these uniform bound conditions may be relaxed when the number of datasets $K$ is fixed. Checking conditions (C2) and (C4) may be done by case by case. Let us look at Example 2 on pages 26-28, where the mean moment condition $g_{k}$ used for parameter estimation is the same over the data sources but outcomes are overdispersed with heterogeneous over-dispersion. It is known that the ana- 
lytic expressions of the moment condition and its derivative are given as follows: $g_{k}(\boldsymbol{W} ; \boldsymbol{\theta})=(1, x)^{T}\left\{y-\exp \left(\theta_{0}+\theta_{1} x\right)\right\}$, and $\partial g_{k}(\boldsymbol{W} ; \boldsymbol{\theta}) / \partial \boldsymbol{\theta}^{T}=$ $-(1, x)^{T}(1, x) \exp \left(\theta_{0}+\theta_{1} x\right)$, respectively, which are continuous in parameters $\theta_{0}$ and $\theta_{1}$, and the same over $k$. Then, it follows that for some $\alpha>2$, with a compact set $\boldsymbol{\Xi}$ under Condition (C1), we have

$$
\begin{aligned}
& \mathbb{E}_{\boldsymbol{\theta}_{0}}\left(\sup _{\boldsymbol{\theta} \in \boldsymbol{\Xi}, 1 \leq k \leq K}\left\|g_{k}\left(\boldsymbol{W}_{k i} ; \boldsymbol{\theta}\right)\right\|_{2}^{\alpha}\right)=\mathbb{E}_{\boldsymbol{\theta}_{0}}\left\{\sup _{\boldsymbol{\theta} \in \boldsymbol{\Xi}}\left[\left(1+X^{2}\right)\left(Y-\exp \left(\theta_{0}+\theta_{1} X\right)\right)^{2}\right]^{\alpha / 2}\right\} \\
& \leq c \sum_{j=0}^{[\alpha+1]} \mathbb{E}_{\boldsymbol{\theta}_{0}}\left\{\left(1+X^{2}\right)^{\alpha / 2} Y^{\alpha-j} \exp \left(c_{1} X\right)^{j}\right\} \\
& \leq \frac{c}{2} \sum_{j=0}^{[\alpha+1]}\left\{\mathbb{E}_{\boldsymbol{\theta}_{0}}\left(Y^{2 \alpha-2 j}\right)+\mathbb{E}_{\boldsymbol{\theta}_{0}}\left[\left(1+X^{2}\right)^{\alpha} \exp \left(c_{1} X\right)^{2 j}\right]\right\}<\infty,
\end{aligned}
$$

where $[a]$ denotes the largest integer smaller than $a, c$ and $c_{1}$ are two positive constants, and the last inequality holds as long as $\mathbb{E}_{\boldsymbol{\theta}_{0}}\left(Y^{j}\right)<\infty$, and $\mathbb{E}_{\boldsymbol{\theta}_{0}}\left(X^{j_{1}} \exp \left(c_{1} X\right)^{j_{2}}\right)<\infty$, for $j, j_{1}, j_{2}=1, \cdots, 2[\alpha+1]$. In Example 2, these two moment conditions automatically hold because $Y$ follows a Poisson distribution for data source \#1, a negative-binomial distribution (resulted from the Poisson-gamma convolution) for data source \#2, and a two-component mixture of Poisson distributions for data source \#3, as well as $X \sim U[-1,1]$. Similarly, under a compact neighborhood $\mathcal{D}$, we have $\mathbb{E}_{\boldsymbol{\theta}_{0}}\left(\sup _{\boldsymbol{\theta} \in \mathcal{D}, 1 \leq k \leq K}\left\|\partial g_{k}\left(\boldsymbol{W}_{k i} ; \boldsymbol{\theta}\right) / \partial \boldsymbol{\theta}^{T}\right\|_{2}\right)=\mathbb{E}_{\boldsymbol{\theta}_{0}}\left(\sup _{\boldsymbol{\theta} \in \mathcal{D}}\left[\left(1+2 X^{2}+X^{4}\right)\right.\right.$ $\left.\left.\times \exp \left(\theta_{0}+\theta_{1} X\right)^{2}\right]^{1 / 2}\right)<\infty$, where the last inequality holds because condition $(\mathrm{C} 1)$ and $X \sim U[-1,1]$. 


\section{Supplementary Materials}

The online supplementary materials contain additional notations, simulation results, and technique details including proofs of theorems.

\section{Acknowledgements}

The authors are grateful to the anonymous reviewers for their constructive comments and suggestions that led to a significant improvement of this paper. Zhou's research was partially supported by Fund of National Natural Science (Nos. 11901470, 11931014 and 11829101) and by Fundamental Research Funds for the Central Universities (Nos. JBK190904 and JBK 1806002). Song's research is supported in part by National Institutes of Health grant R01 ES024732 and National Science Foundation grant DMS1811734.

\section{References}

Boyd, S., Parikh, N., Chu, E., Peleato, B. \& Eckstein, J. (2011), 'Distributed optimization and statistical learning via the alternating direction method of multipliers', Foundations and Trends@ in Machine learning 3(1), 1-122.

Chaudhuri, S., Handcock, M. S. \& Rendall, M. S. (2008), 'Generalized 
REFERENCES36

linear models incorporating population level information: an empiricallikelihood-based approach', Journal of the Royal Statistical Society: Series $B$ (Statistical Methodology) 70(2), 311-328.

Chen, J. \& Qin, J. (1993), 'Empirical likelihood estimation for finite populations and the effective usage of auxiliary information', Biometrika 80(1), 107-116.

Chen, S. \& Kim, J. K. (2014), 'Population empirical likelihood for nonparametric inference in survey sampling', Statistica Sinica 24(1), 335-355.

Citro, C. F. (2014), 'From multiple modes for surveys to multiple data sources for estimates', Survey Methodology 40(2), 137-161.

Efron, B. (1993), 'Bayes and likelihood calculations from confidence intervals', Biometrika $\mathbf{8 0}(1), 3-26$.

Firth, D. (1993), 'Bias reduction of maximum likelihood estimates', Biometrika 80(1), 27-38.

Halevy, A., Rajaraman, A. \& Ordille, J. (2006), Data integration: the teenage years, in 'Proceedings of the 32nd international conference on Very large data bases', VLDB Endowment. 
Han, P. \& Lawless, J. F. (2019), 'Empirical likelihood estimation using auxiliary summary information with different covariate distributions', Statistica Sinica 29, 1321-1342.

Huang, C.-Y., Qin, J. \& Tsai, H.-T. (2016), 'Efficient estimation of the Cox model with auxiliary subgroup survival information', Journal of the American Statistical Association 111(514), 787-799.

Imbens, G. W. (2002), 'Generalized method of moments and empirical likelihood', Journal of Business \& Economic Statistics 20(4), 493-506.

Kovalchik, S. A. (2012), 'Aggregate-data estimation of an individual patient data linear random effects meta-analysis with a patient covariatetreatment interaction term', Biostatistics 14(2), 273-283.

Lenzerini, M. (2002), Data integration: A theoretical perspective, in 'Proceedings of the twenty-first ACM SIGMOD-SIGACT-SIGART symposium on Principles of database systems', ACM.

Liu, D., Liu, R. Y. \& Xie, M. (2015), 'Multivariate meta-analysis of heterogeneous studies using only summary statistics: efficiency and robustness', Journal of the American Statistical Association 110(509), 326-340. 
Lohr, S. L. \& Raghunathan, T. E. (2017), 'Combining survey data with other data sources', Statistical Science 32(2), 293-312.

National Academies of Sciences Engineering and Medicine (2017), 'Federal statistics, multiple data sources, and privacy protection: Next steps', National Academies Press .

Newey, W. K. \& Smith, R. J. (2004), 'Higher order properties of GMM and generalized empirical likelihood estimators', Econometrica 72(1), 219255.

Owen, A. (1991), 'Empirical likelihood for linear models', The Annals of Statistics 19(4), 1725-1747.

Owen, A. B. (1988), 'Empirical likelihood ratio confidence intervals for a single functional', Biometrika 75(2), 237-249.

Owen, A. B. (2001), Empirical Likelihood, Chapman and Hall/CRC.

Qin, J. (2000), 'Combining parametric and empirical likelihoods', Biometrika 87(2), 484-490.

Qin, J. \& Lawless, J. (1994), 'Empirical likelihood and general estimating equations', The Annals of Statistics 22(1), 300-325. 
Qin, J., Zhang, H., Li, P., Albanes, D. \& Yu, K. (2014), 'Using covariatespecific disease prevalence information to increase the power of casecontrol studies', Biometrika 102(1), 169-180.

Simmonds, M. \& Higgins, J. (2007), 'Covariate heterogeneity in metaanalysis: criteria for deciding between meta-regression and individual patient data', Statistics in Medicine 26(15), 2982-2999.

Stout, W. F. (1974), Almost Sure Convergence. Academic Press, New York.

Xie, M.-g. \& Singh, K. (2013), 'Confidence distribution, the frequentist distribution estimator of a parameter: a review', International Statistical Review 81(1), 3-39. 


\section{REFERENCES40}

Table 1: Summary of $\operatorname{Bias}\left(\times 10^{-3}\right), \operatorname{ESE}\left(\times 10^{-3}\right), \operatorname{ASE}\left(\times 10^{-3}\right), \operatorname{CP}(\%)$, and $\operatorname{AIL}\left(\times 10^{-2}\right)$ of $95 \%$ confidence interval as well as average computational time (in seconds) over 2, 000 replicates under 6 cases of sample sizes to estimate the tail probability of Weibull distribution with auxiliary information.

\begin{tabular}{|c|c|c|c|c|c|c|c|c|c|c|}
\hline \multirow[t]{2}{*}{ Method } & BIAS & ESE & ASE & CP(AIL) & Time & BIAS & ESE & ASE & CP(AIL) & Time \\
\hline & \multicolumn{5}{|c|}{$n_{k}=(20,20,20)$} & \multicolumn{5}{|c|}{$n_{k}=(80,80,80)$} \\
\hline Naive & 0.55 & 39.06 & 37.81 & 93.35(14.82) & 0.00 & 0.37 & 19.75 & 19.28 & $92.75(7.56)$ & 0.00 \\
\hline CEL & 2.16 & 26.97 & - & $95.88(10.37)$ & 0.05 & 0.21 & 13.64 & - & $95.65(5.29)$ & 0.08 \\
\hline Meta & -13.99 & 45.18 & 19.65 & $69.77(7.70)$ & 0.32 & -1.72 & 14.64 & 12.94 & $91.20(5.07)$ & 0.34 \\
\hline DOC & 3.18 & 26.43 & - & $96.15(10.39)$ & 4.86 & 0.21 & 13.64 & - & $95.65(5.29)$ & 3.70 \\
\hline \multirow[t]{2}{*}{ SIC } & 7.27 & 27.95 & - & $90.96(9.30)$ & 2.55 & 0.28 & 13.78 & - & $94.49(5.26)$ & 2.35 \\
\hline & \multicolumn{5}{|c|}{$n_{k}=(40,40,40)$} & \multicolumn{5}{|c|}{$n_{k}=(40,80,120)$} \\
\hline Naive & 0.55 & 27.60 & 27.12 & $95.55(10.63)$ & 0.00 & 0.37 & 19.75 & 19.28 & $92.75(7.56)$ & 0.00 \\
\hline CEL & 0.27 & 19.73 & - & $94.19(7.43)$ & 0.06 & 0.21 & 13.64 & - & $95.65(5.29)$ & 0.08 \\
\hline Meta & -4.62 & 25.02 & 17.17 & $83.35(6.73)$ & 0.31 & -2.20 & 16.51 & 12.86 & $89.51(5.04)$ & 0.35 \\
\hline DOC & 0.37 & 19.59 & - & $94.38(7.43)$ & 3.91 & 0.21 & 13.64 & - & $95.65(5.29)$ & 3.93 \\
\hline \multirow[t]{2}{*}{ SIC } & 0.59 & 20.45 & - & $91.67(7.18)$ & 2.35 & 0.40 & 13.82 & - & $94.48(5.24)$ & 2.54 \\
\hline & \multicolumn{5}{|c|}{$n_{k}=(150,150,150)$} & \multicolumn{5}{|c|}{$n_{k}=(80,150,220)$} \\
\hline Naive & 0.51 & 14.34 & 14.13 & $93.30(5.54)$ & 0.00 & 0.51 & 14.34 & 14.13 & $93.30(5.54)$ & 0.00 \\
\hline CEL & 0.29 & 9.94 & - & $94.90(3.88)$ & 0.10 & 0.29 & 9.94 & - & $94.90(3.88)$ & 0.10 \\
\hline Meta & -0.64 & 10.32 & 9.69 & $93.30(3.80)$ & 0.42 & -0.73 & 10.41 & 9.69 & $92.75(3.80)$ & 0.42 \\
\hline DOC & 0.30 & 9.94 & - & $94.95(3.88)$ & 3.26 & 0.29 & 9.94 & - & $94.95(3.88)$ & 3.43 \\
\hline SIC & 0.37 & 10.00 & - & $94.50(3.86)$ & 2.63 & 0.30 & 10.04 & - & $94.30(3.86)$ & 2.78 \\
\hline
\end{tabular}




\section{REFERENCES41}

Table 2: Summary of $\operatorname{BIAS}\left(\times 10^{-2}\right), \operatorname{ESE}\left(\times 10^{-2}\right), \operatorname{ASE}\left(\times 10^{-2}\right), \operatorname{CP}(\%)$ and $\operatorname{AIL}\left(\times 10^{-1}\right)$ of $95 \%$ confidence interval, relative interval length compared to that of the CEL method (RIL), and average computation time (in seconds) over 2,000 replicates under different sample sizes for estimating the slope parameter $\beta_{1}$ in a log-linear model with over-dispersion.

\begin{tabular}{|c|c|c|c|c|c|c|c|c|}
\hline$n_{k}$ & method & BIAS & ESE & ASE & $\mathrm{CP}$ & AIL & RIL & Time \\
\hline \multirow[t]{5}{*}{$(50,100,150)$} & GLM & -0.18 & 17.34 & 10.24 & 74.55 & 4.01 & 0.58 & 0.00 \\
\hline & CEL & -0.18 & 17.34 & - & 94.80 & 6.88 & 1.00 & 0.88 \\
\hline & Meta & -0.75 & 14.36 & 13.04 & 93.33 & 5.11 & 0.74 & 0.14 \\
\hline & $\mathrm{DOC}$ & -0.19 & 17.31 & 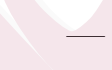 & 94.80 & 6.88 & 1.00 & 7.90 \\
\hline & SIC & 0.67 & 14.41 & - & 93.50 & 5.20 & 0.76 & 6.20 \\
\hline \multirow[t]{5}{*}{$(100,200,300)$} & GLM & 0.14 & 12.43 & 7.21 & 74.00 & 2.83 & 0.58 & 0.00 \\
\hline & CEL & 0.14 & 12.43 & - & 94.75 & 4.86 & 1.00 & 1.02 \\
\hline & Meta & -0.38 & 9.77 & 9.45 & 94.20 & 3.70 & 0.76 & 0.18 \\
\hline & DOC & 0.13 & 12.39 & - & 94.75 & 4.86 & 1.00 & 7.57 \\
\hline & $\mathrm{SIC}$ & 0.01 & 9.76 & - & 94.60 & 3.75 & 0.77 & 6.42 \\
\hline \multirow[t]{5}{*}{$(200,400,600)$} & GLM & 0.17 & 8.91 & 5.09 & 73.20 & 1.99 & 0.58 & 0.01 \\
\hline & CEL & 0.17 & 8.91 & - & 94.50 & 3.44 & 1.00 & 1.55 \\
\hline & Meta & -0.25 & 6.95 & 6.76 & 94.35 & 2.65 & 0.77 & 0.25 \\
\hline & DOC & 0.17 & 8.88 & - & 94.50 & 3.44 & 1.00 & 10.54 \\
\hline & SIC & -0.43 & 6.96 & - & 94.60 & 2.68 & 0.78 & 8.79 \\
\hline
\end{tabular}




\section{REFERENCES42}

Table 3: Summary of results for estimating $\beta_{3}$ in a log-linear model. Different scenarios of imbalance in covariates' distribution are given by various probabilities $p$ 's, and S0 represents an extremely unbalanced situation.

\begin{tabular}{|c|c|c|c|c|c|c|c|c|c|c|}
\hline $\mathrm{p}$ & 0.5 & 0.2 & 0.1 & 0.05 & S0 & 0.5 & 0.2 & 0.1 & 0.05 & S0 \\
\hline & \multicolumn{5}{|c|}{ Centralized GLM } & \multicolumn{5}{|c|}{ Centralized EL } \\
\hline BIAS & 0.00 & -0.00 & -0.00 & -0.01 & -0.00 & 0.00 & -0.01 & -0.00 & -0.01 & -0.00 \\
\hline ESE & 0.07 & 0.08 & 0.11 & 0.16 & 0.06 & 0.07 & 0.08 & 0.11 & 0.16 & 0.06 \\
\hline $\mathrm{CP}$ & 94.45 & 94.65 & 94.75 & 95.65 & 94.85 & 94.60 & 94.30 & 92.85 & 92.33 & 94.70 \\
\hline AIL & 0.26 & 0.32 & 0.44 & 0.62 & 0.22 & 0.25 & 0.32 & 0.43 & 0.59 & 0.22 \\
\hline Time & 0.00 & 0.00 & 0.00 & 0.00 & 0.00 & 30.12 & 30.89 & 30.86 & 33.35 & 23.37 \\
\hline $\mathrm{PAC}$ & 1.00 & 1.00 & 1.00 & 1.00 & 1.00 & 1.00 & 1.00 & 1.00 & 0.99 & 1.00 \\
\hline
\end{tabular}

Distributed EL

$\begin{array}{lrrrrrr} & & & & & & \\ \text { BIAS } & 0.00 & -0.00 & -0.00 & -0.01 & -0.00 \\ \text { ESE } & 0.07 & 0.08 & 0.11 & 0.16 & 0.06 \\ \text { CP } & 94.55 & 94.30 & 92.83 & 92.60 & 94.67 \\ \text { AIL } & 0.25 & 0.32 & 0.43 & 0.59 & 0.22 \\ \text { Time } & 29.47 & 19.12 & 14.43 & 20.66 & 39.02 \\ \text { PAC } & 1.00 & 1.00 & 1.00 & 1.00 & 0.99\end{array}$

\begin{tabular}{|c|c|c|c|c|c|c|c|c|c|c|}
\hline \multirow[b]{2}{*}{ BIAS } & \multicolumn{5}{|c|}{ Meta GLM } & \multicolumn{5}{|c|}{ Meta EL } \\
\hline & -0.01 & 0.00 & 0.02 & 0.02 & - & -0.01 & 0.01 & - & - & - \\
\hline ESE & 0.07 & 0.08 & 0.11 & 0.19 & - & 0.08 & 0.09 & - & - & - \\
\hline $\mathrm{CP}$ & 94.30 & 94.80 & 95.05 & 81.82 & - & 79.61 & 78.21 & - & - & - \\
\hline AIL & 0.26 & 0.32 & 0.43 & 0.54 & - & 0.20 & 0.22 & - & - & - \\
\hline Time & 0.02 & 0.02 & 0.02 & 0.02 & - & 5.83 & 6.32 & - & - & - \\
\hline $\mathrm{PAC}$ & 1.00 & 0.97 & 0.42 & 0.01 & 0.00 & 0.88 & 0.04 & 0.00 & 0.00 & 0.00 \\
\hline
\end{tabular}


Table 4: Results of logistic regression with the SRTR data by the methods of distributed empirical likelihood (DEL) method, Meta method (Meta2), region-specific maximum likelihood estimation on Alaska (GLM_AK) and on Wyoming (GLM_WY).

\begin{tabular}{lcccccccc}
\hline \hline & \multicolumn{2}{c}{ DEL } & \multicolumn{2}{c}{ Meta2 } & \multicolumn{2}{c}{ MLE_AK } & \multicolumn{2}{c}{ MLE_WY } \\
& EST & $p$-val & EST & $p$-val & EST & $p$-val & EST & $p$-val \\
& & $\left(\times 10^{-1}\right)$ & & $\left(\times 10^{-1}\right)$ & & $\left(\times 10^{-1}\right)$ & & $\left(\times 10^{-1}\right)$ \\
\hline Intercept & -1.94 & 0.01 & -1.77 & 0.01 & -1.96 & 0.22 & -1.83 & 0.09 \\
rec_sex & 0.55 & 0.14 & 0.42 & 1.19 & 0.67 & 1.17 & 0.38 & 2.69 \\
don_sex & -0.57 & 0.16 & -0.48 & 0.65 & -1.13 & 0.07 & -0.20 & 5.57 \\
rec_age & -0.01 & 0.18 & -0.01 & 1.31 & -0.01 & 5.02 & -0.02 & 1.46 \\
don_age & 0.00 & 1.37 & 0.00 & 9.64 & -0.01 & 7.04 & 0.01 & 7.21 \\
rec_bmi & 0.19 & 1.02 & 0.26 & 3.45 & 0.13 & 7.45 & 0.49 & 2.03 \\
don_bmi & -0.52 & 0.45 & -0.30 & 3.90 & -0.94 & 1.34 & -0.10 & 8.17 \\
prev_tx & 0.43 & 0.86 & 0.33 & 3.53 & 1.00 & 0.51 & -0.18 & 7.29 \\
rec_hcv & -0.34 & 1.20 & -0.24 & 7.47 & -0.49 & 6.53 & -0.17 & 8.72 \\
\hline \hline
\end{tabular}

